Macedonian Pharmaceutical Bulletin, 66 (Suppl 1) 79 - 80 (2020)

Online ISSN $1857-8969$

UDC: $614.253 .8(497.7)(083.86)$

DOI: 10.33320/maced.pharm.bull.2020.66.03.039

Short communication

\title{
Patient registries in regulatory decision making - a survey of Macedonian registries
}

\author{
Elona Chilku ${ }^{1}$, Goran Kochinski ${ }^{2}$, Kristina Mladenovska $^{3}$ \\ ${ }^{1}$ Agency for Medicines and Medical Devices, Cyril and Methodius 54, 1000 Skopje, N. Macedonia \\ ${ }^{2}$ Ministry of Health, e-Health Directorate, 50 Divizija 14-a, 1000 Skopje, N. Macedonia \\ ${ }^{3}$ Faculty of Pharmacy, Ss. Cyril and Methodius University, Mother Theresa 47, 1000 Skopje, N. Macedonia
}

\section{Introduction}

Patient registries are "organized systems that use observational methods to collect uniform data on a population defined by a particular disease, condition, or exposure, and that is followed over time" (EMA, 2017). According to ISPOR, a registry includes a "prospective observational study of subjects with certain shared characteristics, which collects ongoing and supporting data over time on welldefined outcomes of interest for analysis and reporting" (Polygenis et al., 2013). The data collected help policy makers, researchers, healthcare professionals and many others to prioritize their activities and actions and plan restricted resources in areas of their responsibilities. Establishing and governing a patient registry requires use of standardized methodologies, processes and technologies that will enable researchers, health technology assessment organizations and policy creators to compare, analyze and use data from different (inter)national registries for extracting new knowledge and make informed decisions about individual patients and or entire populations. In this paper, the results from the survey of patient registries established and planned in the Republic of N. Macedonia (RoM) are presented. In addition, an overview of funding and legislation concerning registry set-up, sustainability, data protection and reuse is given.

\section{Materials and Methods}

To collect the information on national patient registries, a questionnaire was used, consisted of questions related to the primary propose of the registry, data collection, handling, funding and governing. Some of the stakeholders had been interviewed in person or by phone. The target groups were government institutions, academia, clinical centers and hospitals, professional and patient associations, and marketing authorization holders.

\section{Results and Discussion}

Thirty-five registries were foreseen at national level. Out of them, 22 are disease/condition based (registries of patients having the same diagnosis or the same group of conditions), 10 are health service based registries (consisted of patients having a common procedure, clinical encounter or hospitalization) and one is registry based on product (patients exposed to biopharmaceutical products, medical devices or diagnostic/therapeutic equipment), while 3 are not patient registries, but registries of healthcare professionals and institutions. In disease/condition category several subcategories

*elonachi@gmail.com 
have been recognized based on organ system or clinical field, with the largest number (4) falling under the cancer/tumor (integrated in one Cancer Registry) and injuries/accidents subcategories, followed by infectious (3), coronary/vascular (2), congenital (2), substance abuse/addiction (2), renal/ urogenital (1), disabilities (1), mental/psychiatric (1) and diabetes/metabolic/endocrine (1) subcategories. Rare diseases are incorporated in one register only. The service-based patient registries are divided into the following subcategories: obstetric and gynecological services (births, abortions, medically assisted fertilization) (3), preventative services, quality of care and health monitoring (3), registries of donors (blood and organ) (2) and causes of deaths (2). The Insulin Registry is the one based on product.

Out of 35 foreseen patient registries, 10 registries are already established and active in electronic form, 3 are expected to be activated during 2020/2021 and 10 are in process of preparation. Comparing to the active registers across the European countries (data from 2015), the number of active registers is lower than the one in Austria and Italy (38 each), Finland, Sweden, Croatia, Poland, Norway and Slovenia (between 32 and 15), but higher than in the neighboring countries, Greece and Serbia (two each) and Albania and Bulgaria (one each) (Zaletel and Kralj, 2015).

The registries in the RoM are dominantly governed by the Ministry of Health. They contain information from all institutions in which patients are diagnosed and treated for the corresponding disease or the healthcare services are provided. Thus, all national registries are funded by national government authority and there is "no specific funding" such as an umbrella organization, certain project, etc. The Institute of Public Health uses these data for health care planning, incl. primary prevention, diagnosis and treatments. Because of the limitations of premarketing clinical trials (short duration, small sample size, narrowly defined population, limited comparison groups, etc.), observation of medical products after their approval for marketing is important, especially for products granted conditional marketing authorization, since registries may provide data to confirm their safety and/or effectiveness. However, the registries in RoM are still not extensively used for benefit/risk evaluation of medicines.

Most of the registries are based on electronic health care records, although paper based questionnaires/health records/laboratory results are still used for data collection. As literature data point out, almost half of the EU registries are still based on paper-and-pen mode, which "causes lower data quality, it is costly and time consuming and does not allow any control of the data filled in" (Zaletel and Kralj, 2015). The set-up, sustainability, data protection and re-use of patient registries are regulated by the Law on medical records (Official Gazette of RM 20/09) and the Rulebook on how to access, distribute, publish, use, store and protect data from the integrated health information system (http://zdravstvo.gov.mk). Insufficient information on quality standards and no quality control/assessment tools for the registries in the RoM was available. The data contained in the registries are available to researchers from other institutions and there are protocols that enable them to access the data.

\section{Conclusion}

The number and subcategories of patient registries in the RoM comparing to the European countries is satisfactory. However, awareness of quality standards should be increased and quality assessment tools introduced. As patient registers are opened for research purposes, their extensive utilization for post-marketing evaluation of medicines should be also considered.

\section{References}

European Medicines Agency, 2017. Patient Registry Initiative - Strategy and Mandate of the CrossCommittee Task Force. EMA/180341/2017.

Polygenis, D., Frame, D., Blanchette, C., Gemmen, E., Kamble, S., Larson, L., Molsen, E., 2013. Eds. ISPOR Taxonomy of Patient Registries: Classification, Characteristics and Terms. Lawrenceville, NJ.

Zaletel, M., Kralj, M., 2015. Patient registries in Methodological guidelines and recommendations for efficient and rational governance of patient registries. ISBN 978-961-6911-75-7 (pdf). 